\title{
Influence of the Drive Plate with Different Surface Textures on the Property of Carbonyl Iron-Based Magnetorheological Fluid
}

\author{
He Lu $\mathbb{D}^{1,2}$ Lifeng Wang, ${ }^{3}$ Lili Dai, ${ }^{1}$ Hongxing Ding $\mathbb{D}^{1},{ }^{1}$ Qiuxiang Zhang, ${ }^{2}$ \\ and Xinhua Liu $\mathbb{D}^{2}$ \\ ${ }^{1}$ Lianyungang Normal College, Lianyungang 222006, China \\ ${ }^{2}$ School of Mechatronic Engineering, China University of Mining and Technology, Xuzhou 221116, China \\ ${ }^{3}$ Chongqing Three Gorges University, Chongqing 404000, China
}

Correspondence should be addressed to He Lu; luhe119879@126.com and Hongxing Ding; dinghxing@126.com

Received 31 May 2020; Revised 22 July 2020; Accepted 25 July 2020; Published 16 October 2020

Academic Editor: Gloria Terenzi

Copyright (c) $2020 \mathrm{He}$ Lu et al. This is an open access article distributed under the Creative Commons Attribution License, which permits unrestricted use, distribution, and reproduction in any medium, provided the original work is properly cited.

Aiming to study the effect of drive plate with different surface topographies on the wear property of magnetorheological fluid (MRF), some specific experiments are carried out and analyzed in this paper. Firstly, experiment materials and test methods for MRF are elaborated, and four different surface topography drive plates are designed and manufactured. MRF test-bed is built up to test the rheological properties of MRF. Moreover, the surface morphology of particles is taken using a scanning electron microscope (SEM). Finally, experimental results show that there are remarkable influences on tribological properties of carbonyl iron-based MRF with different drive plates. Therefore, the drive plate with specific surface topography can meet the needs of different MRF transmission systems.

\section{Introduction}

As a kind of smart material that is sensitive to magnetic field, magnetorheological fluid (MRF) is a solid-liquid two-phase system formed by micron or submicron magnetic particles and additives disperse in a nonmagnetic carrier liquid $[1,2]$. MRF changes into a solid-like structure from a liquid-like structure when exposed to an external magnetic field. When the external magnetic field is removed, the behavior of MRF reverts to the previous state within a few milliseconds. Such phenomenon is called magnetorheological effect [3, 4]. As magnetorheological effect is rapid, continuous, and reversible, MRF is widely applied in biological medicine [5], automobile industry [6], polishing technology [7, 8], aerospace [9], mechanical engineering, and other fields [10, 11].

In practical engineering applications, carbonyl ironbased magnetorheological fluid with different physical parameters has different application properties, which has received extensive research by scholars. The corrosion process of carbonyl iron particles on magnetorheological behavior was investigated by Plachy et al., indicating that carbonyl iron particles were oxidized at fierce thermal oxidation at $500^{\circ} \mathrm{C}$ in the air and mild chemical oxidation in $0.05 \mathrm{M} \mathrm{HCl}$ [12]. Sedlacik and Pavlinek performed an experimental investigation to elucidate the effect of partial substitution on the overall MR performance as well as sedimentation stability; the sedimentation test showed positive role of dimorphic composition of dispersed phase on the sedimentation stability [13]. A systematical study of the overall influence of carbon allotrope additives on performance, stability, and redispersibility of magnetorheological fluids was carried out by Cvek et al., showing that carbon nanotubes had a better effect on stability and redispersibility of MRF [14]. In addition, the effect of acid additives on the stability and rheological properties of a suspension of carbonyl iron (CI) microparticles dispersed in silicone oil was studied by Ashtiani and Hashemabadi. Experimental results showed that, by increasing carbon chain length of acids, yield stress and stability increased up to 22 times (at $H=362 \mathrm{kA} / \mathrm{m}$ ) and 7 times, respectively [15]. The relationship between shear stress and volume fraction and shear rate of MRFs was studied by Sun et al., and it was 
found that the volume fraction had great effect on the yield stress of MRF [16].

Particle morphology also has great influence on the properties of magnetorheological fluids. The characterization, magnetic, and rheological properties of plate-like carbonyl iron particle (CIP) in comparison with conventional spherical CIP were studied by Shilan et al., and they indicated that the plate-like CIP obtained higher saturation magnetization (about $8 \%$ ) than that of the spherical particles [17]. Moreover, a nonspherically shaped iron particle-based magnetorheological (MR) fluid, particularly flake-shaped, is synthesized to evaluate the performance of an MR brake by Patel et al., and it was shown that flake-shaped particle-based MR fluid with $70 \%$ weight fraction of iron particles exhibits $17 \%$ higher breaking torque at relatively low magnetic field strength compared to spherically shaped MR fluid with $72 \%$ particle weight fraction [18]. Effect of nanocelluloses on the magnetoresponsive behavior and stability of MR fluids was studied by Wang et al., and they indicated that both CNC and CNF can stabilize MR fluids and improve their sensitivity to alterations of magnetic field strengths [19]. The effect of nanodiamond on the MRF was studied by Zhao et al., and they demonstrated that the physical properties and external working conditions of the nanodiamond could have a higher impact on MRF, which was of high significance to the preparation of MRF with excellent performance [20]. The iron nanoparticles and commercial carbonyl iron microsized particles were used in the dispersing phase to prepare MR fluids; magnetorheological effect and sedimentation stability were measured for comparison by $\mathrm{Zhu}$ et al., and they indicated that the iron nanoparticles-based MR fluids present a slightly lower MR effect but much better sedimentation stability with respect to the MR fluids with carbonyl iron microsized particles [21].

Furthermore, external working condition is also one of the key factors affecting the properties of MRF. The temperature effect on performance of compressible magnetorheological fluid suspension systems was studied by McKee et al. and it was found that the shear yield stress of the magnetorheological fluid remained unchanged within the testing range, while both the plastic viscosity, using the Bingham plastic model, and the bulk modulus of the magnetorheological fluid decreased as the temperature of the fluid increased [22]. The microscopic characteristics of a magnetorheological fluid (MRF) in a magnetic field was studied by Wang et al., and they indicated that the chain structure of the same MRF becomes more apparent as the magnetic field strength increases and, in the same external magnetic field, the chain structure also becomes more apparent with an increase in the particle volume fraction [23].

Bearing the above observations in mind, the research of $\mathrm{MRF}$ is greatly in the component parameters of MRF (magnetic particles, carrier fluid, and additives) and external conditions, such as magnetic field and temperature. However, few studies focus on surface texture of the drive plate on the property of MRF. In this paper, MRF test-bed is used to study the effects of different surface topography on properties of carbonyl iron-based magnetorheological fluid. Sedimentation stability, zero-field viscosity, maximum transmittable torque, and shear yield stress of the samples have been studied and analyzed. Test results show that there are remarkable influences on tribological properties of carbonyl iron-based MRF with different surface texture.

The rest of this paper is organized as follows: experimental methods and preparation of the MRF are elaborated in Section 2. Results and discussion based on drive plate with different surface texture on MRF experiments are discussed in Section 3. Our conclusions and future work are summarized in Section 4.

\section{Experiment}

2.1. Preparation of $M R F$. Carbonyl iron particles and synthesized base oil are used as magnetic particles and carrier fluid for MRF, respectively. Carbonyl iron powders consisted of polydisperse spherical particles with diameter ranging between 1 and $2 \mu \mathrm{m}$. Due to its excellent hightemperature and low-temperature performance and wide range of working temperatures, PAO 6 (Poly-Alpha-Olefins 6 ) is chosen as carrier fluid. Firstly, the synthesized base oil is heated from normal temperature to $60^{\circ} \mathrm{C}$ by a digital magnetic agitator. Secondly, dispersant, thickener, and activator are added to the synthesized base oil with certain proportion successively. Thirdly, the mixture is magnetically stirred for about 2 hours until it is well blended and the speed is kept at $300 \mathrm{rpm}$; then we get compound liquid. Fourthly, a certain percentage of carbonyl iron powder is added to the compound liquid gradually. Fifthly, stirring is performed and mechanical stirring speed is kept at $1800 \mathrm{rpm}$ for about 8 hours. Sixthly, the suspension liquid is stirred at $25^{\circ} \mathrm{C}$ for about 1 hour. MRF of $30 \%$ mass fraction is prepared by the above method. The preparation process of MRF is shown in Figure 1. Then we get the prepared carbonyl iron-based magnetorheological fluid sample that is marked as MRF-0.

2.2. Experimental Methods for Properties of MRF. As shown in Figure 2, there are three working modes of MRF device: flow mode, shear mode, and squeeze mode. MRF transmission device is based on the shear mode. According to the model of Newton fluid and Bingham fluid, the relation between total shear stress $\tau$ and shear strain rate $\dot{\gamma}$ is given by [24]

$$
\begin{aligned}
& \tau=\tau_{y}(B) \operatorname{sgn}(\dot{\gamma})+\eta \dot{\gamma}, \quad \tau>\tau_{(y)} B, \\
& \dot{\gamma}=0, \quad \tau \leq \tau_{(y)} B,
\end{aligned}
$$

where $\tau_{y}(B)$ is the yield stress in response to the applied magnetic field intensity $B$ and $\eta$ is the constant plastic viscosity, which is the apparent viscosity.

Before the magnetic particles are not magnetized to saturation, $\tau_{y}(B)$ increases with the increasing magnetic field intensity $B$ and it is given by [24]

$$
\tau_{y}(B)=\alpha B^{n},
$$

where $B$ is the applied magnetic flux density and $\alpha$ and $n$ are constant parameters that approximate the relationship 


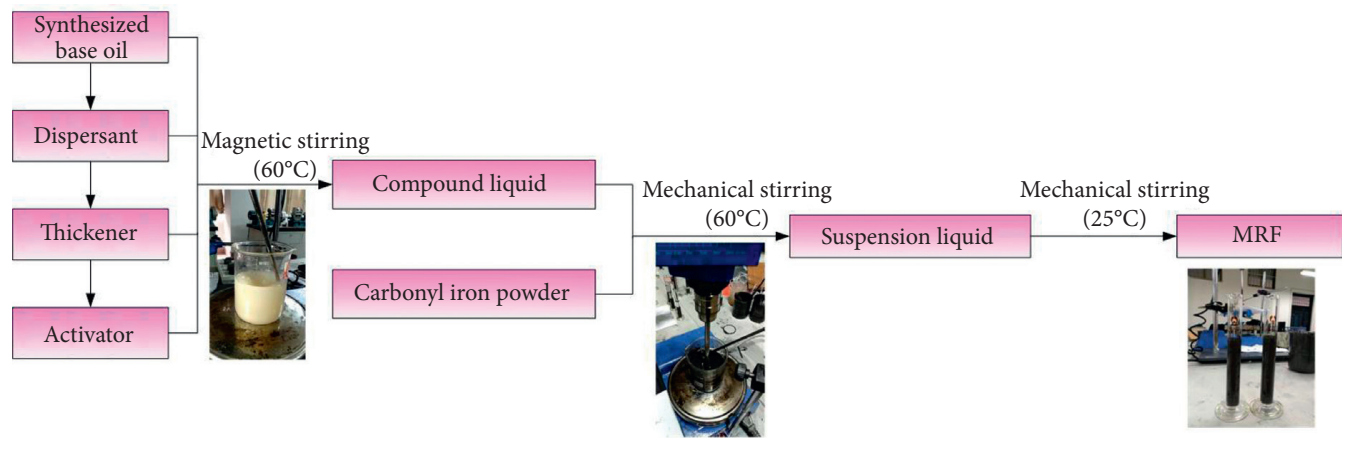

FIgURE 1: Preparation process of MRF.

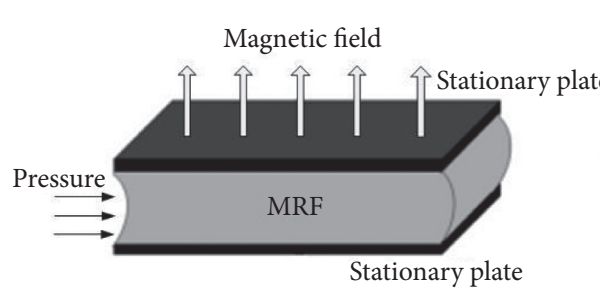

Flow mode

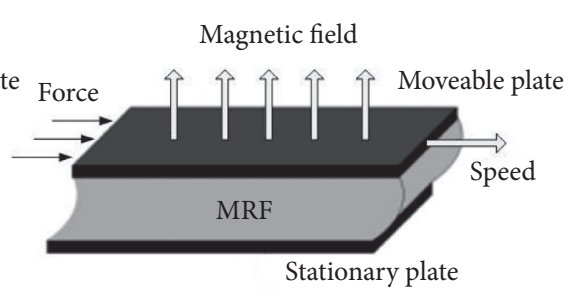

Shear mode

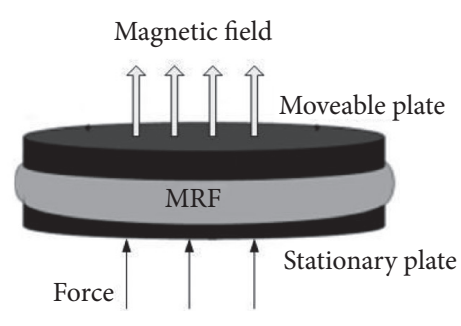

Squeeze mode

FIGURE 2: Working modes of MRF device.

between the magnetic field intensity and the yield stress for MRF, which is determined by the properties of MRF.

In this paper, a disc rotary shear test device is used to test the transferable torque and its working principle is shown in Figure 3, and the resistant torque can be written as [24]

$$
T=2 \pi \int_{r_{1}}^{r_{2}} \tau r^{2} \mathrm{~d} r .
$$

The angular velocities of the rotating disks are $\omega_{1}$ and $\omega_{2}$, and $h$ is the thickness of the MR fluid gap; then

$$
\dot{\gamma}=\frac{r\left(\omega_{1}-\omega_{2}\right)}{h} \text {. }
$$

Substituting equations (1)-(5), the resistant torque is given by

$$
T=\int_{r_{1}}^{r_{2}} 2 \pi r^{2} \mathrm{~d} r=\frac{2 \pi \tau_{y}}{3}\left(r_{2}^{3}-r_{1}^{3}\right)+\frac{\pi \eta \Delta \omega}{2 h}\left(r_{2}^{4}-r_{1}^{4}\right) .
$$

It can be seen from the above equation that the resistant torque of MRF transmission device is mainly composed of two parts, $T_{B}=\left(2 \pi \tau_{y} / 3\right)\left(r_{2}^{3}-r_{1}^{3}\right)$ and $T_{\eta}=(\pi \eta \Delta \omega / 2 h)\left(r_{2}^{4}-r_{1}^{4}\right)$. $T_{B}$ is produced by shearing chain columnar structure of MRF, and $T_{\eta}$ is formed by the viscidity of MRF that is related to physical property of MRF.

In order to observe the dynamic evolution process of magnetorheological fluid with the applied magnetic field, MRF microstructure observation device is built up (Figure 4). As shown in Figure 5, magnetic particles are randomly distributed in carrier liquid without an applied magnetic field. The magnetic particles attract each other and line up into chains in the direction of an applied magnetic field and the chains are wider and longer with the intensity of magnetic field increasing.
A testing device is designed and its integral structure is shown in Figure 6. In order to study the effects of different surface topography on properties of carbonyl iron-based magnetorheological fluid, drive plate with smooth surface, ring groove, radial groove, and pitted surface are designed, where the depth of the grooves and pitted surface is $2 \mathrm{~mm}$, as shown in Figure 7. MRF test-bed is designed and constructed, as shown in Figure 8. Firstly, four samples of MRF with the same composition are prepared as a reference sample before the experiment. Sedimentation stability, zerofield viscosity, and shear yield stress are tested, respectively. Secondly, the sample of MRF is injected into the MRF working gap of the testing device. Thirdly, loads of magnetic powder brake are kept at $20 \mathrm{~N} / \mathrm{m}$, regulating DC electrical source and delivering up to $3.5 \mathrm{~A}$ of continuous output current, running the system. Fourthly, the maximum load is recorded once an hour, which can be transferred by the test device when the systems stabilize. Fifthly, the system is stopped when the load remains constant, and samples are taken to test its dispersion stability, zero-field viscosity, and shear yield stress. Sixthly, drive plate is changed with four different surface topographies shown in Figure 2, and steps 2 through 5 are repeated.

\section{Results and Discussion}

MRF samples' index and the corresponding test drive plate in the wear experiments are given in Table 1.

3.1. SEM Images of Magnetic Particles Morphology. The surface morphology of magnetic particles is observed by scanning electron microscopy (SEM). Carbonyl iron particles are essentially spheroids, as shown in Figure 9(a). The 

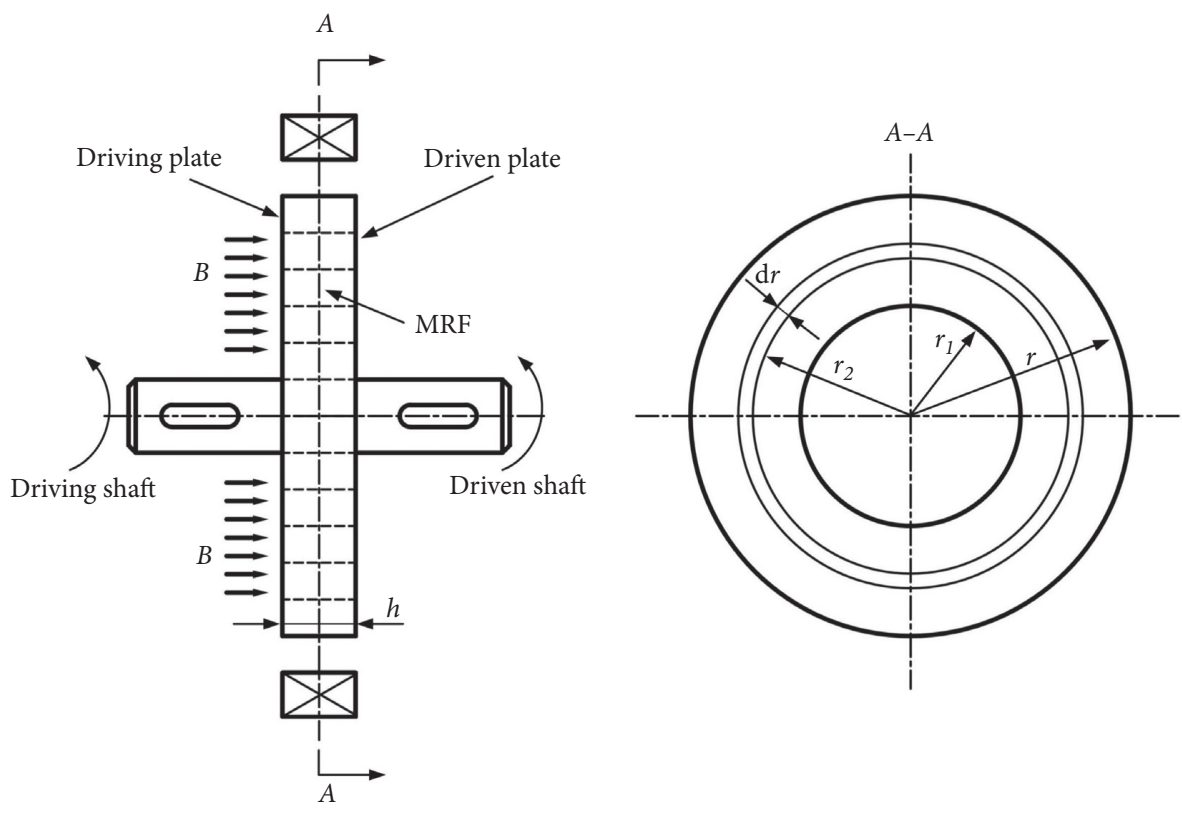

FIGURE 3: Working principle of MRF shear.

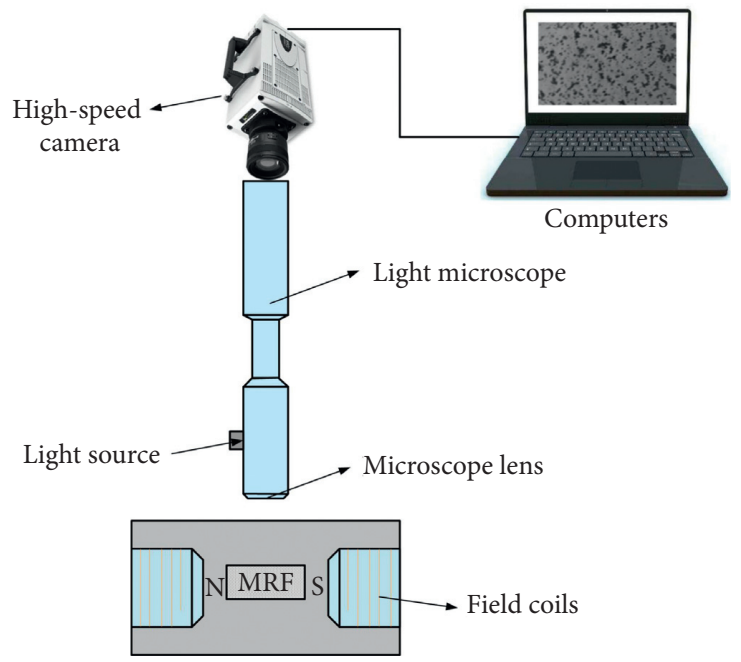

FIGURE 4: Diagram of MRF microstructure observation device.

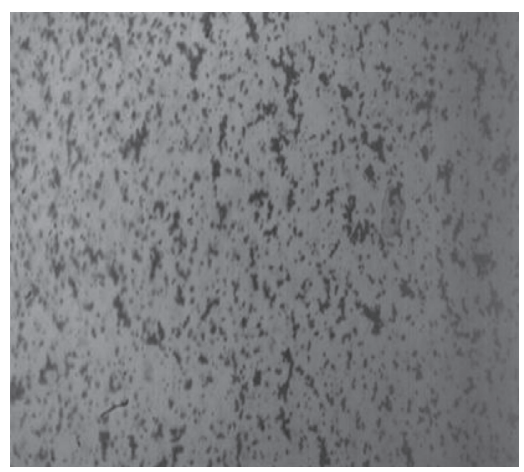

$H=0 \mathrm{~A} / \mathrm{m}$

(a)

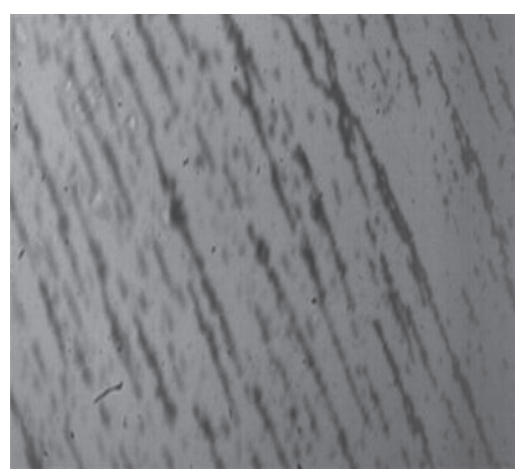

$H=10^{-2} \mathrm{~A} / \mathrm{m}$

(b)

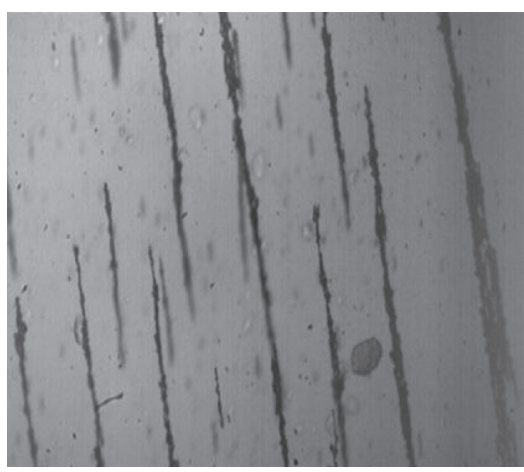

$H=2 \times 10^{-2} \mathrm{~A} / \mathrm{m}$

(c)

FIgURE 5: Microstructure of magnetorheological fluid under different magnetic intensity. 


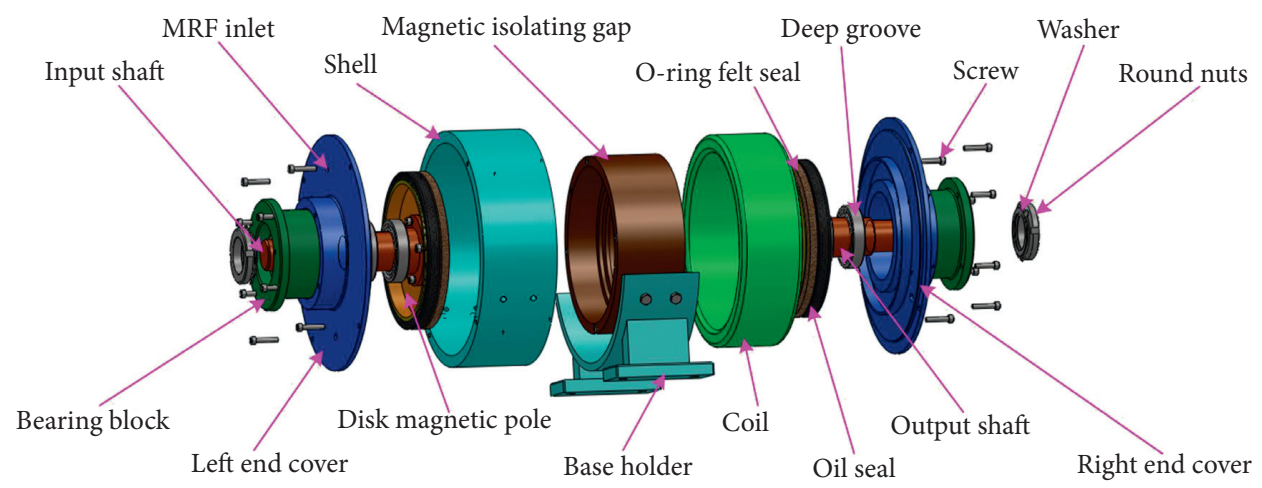

Figure 6: Explosive view of testing device.

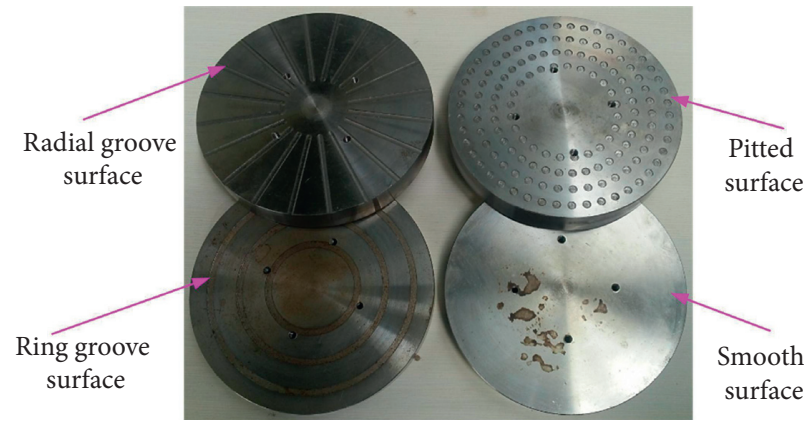

FIgURE 7: Drive plate with different surface topography.

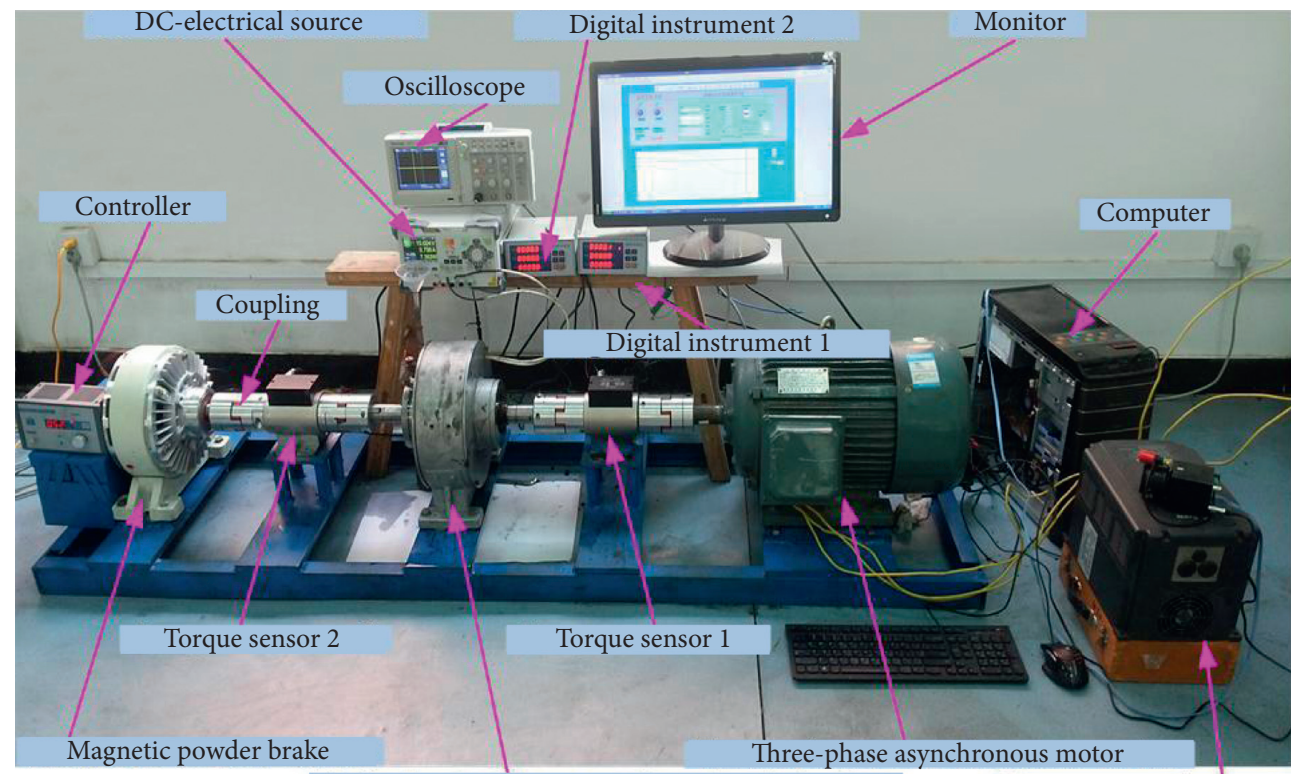

Tribological properties test device

Frequency changer

FIGURE 8: MRF test-bed.

modified particles with floccule surface can improve the suspension stability of MRF shown in Figure 9(b). Figures 9(c) and 9(d) show that particles are worn after the wear test due to the interactions between the particles $[25,26]$.
3.2. Sedimentation Test. The sedimentation stability of the samples is investigated by visual observation method and can be expressed by sedimentation ratio $V$ [27]:

$$
V=\frac{h}{H} \times 100 \% .
$$


TABLE 1: Index of MRF samples and the corresponding test drive plate.

\begin{tabular}{lcc}
\hline Index & Experimental projects & Surface morphology of test drive plate \\
\hline MRF-0 & - & - \\
\hline MRF-1 & Redimentation stability & groove surface \\
MRF-2 & Pitted surface \\
MRF-3 & Smooth surface \\
MRF-4 & Zero-field viscosity & Radial groove surface \\
\hline MRF-1 & & Ring groove surface \\
MRF-2 & & Pitted surface \\
MRF-3 & Smooth surface \\
MRF-4 & & Radial groove surface \\
\hline MRF-1 & Ring groove surface \\
MRF-2 & Pitted surface \\
MRF-3 & Smooth surface \\
MRF-4 & & Radial groove surface \\
\hline MRF-1 & & Ring groove surface \\
MRF-2 & Phitted surface \\
MRF-3 & & Smooth surface \\
MRF-4 & & Radial groove surface \\
\hline
\end{tabular}

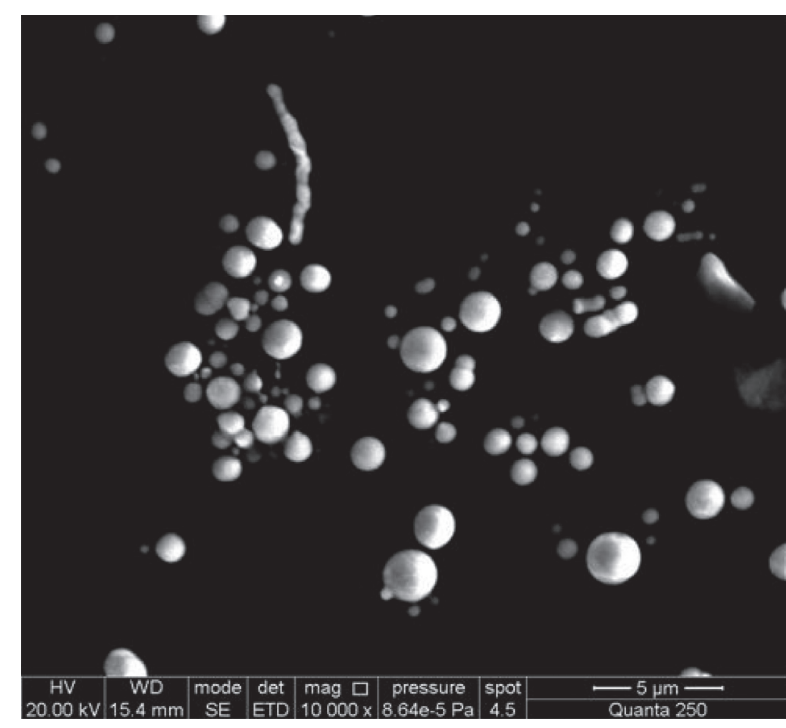

(a)

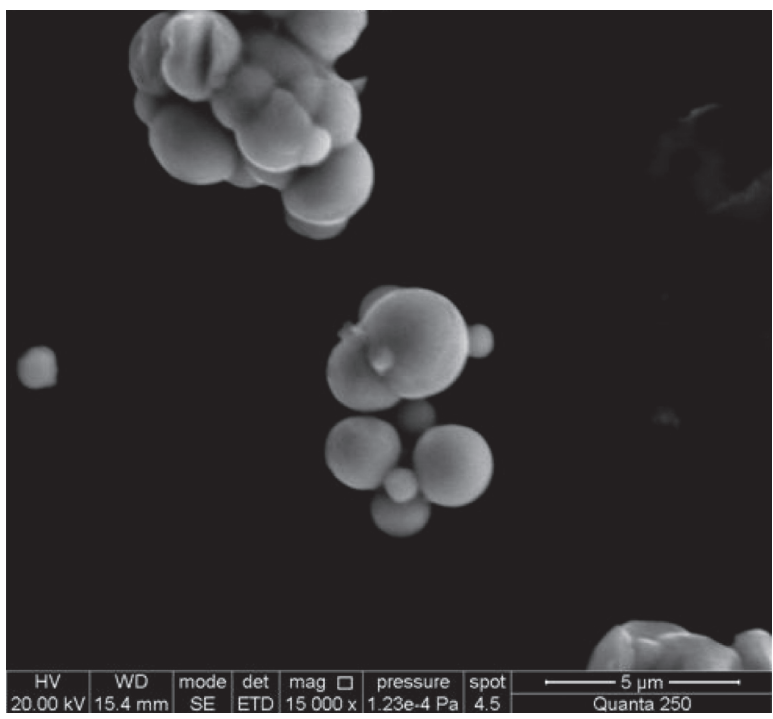

(c)

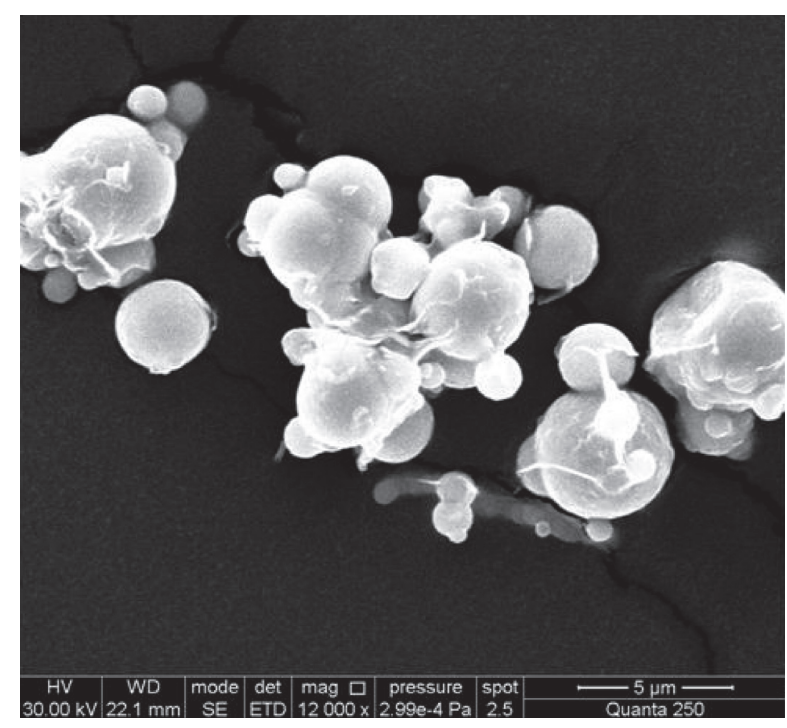

(b)

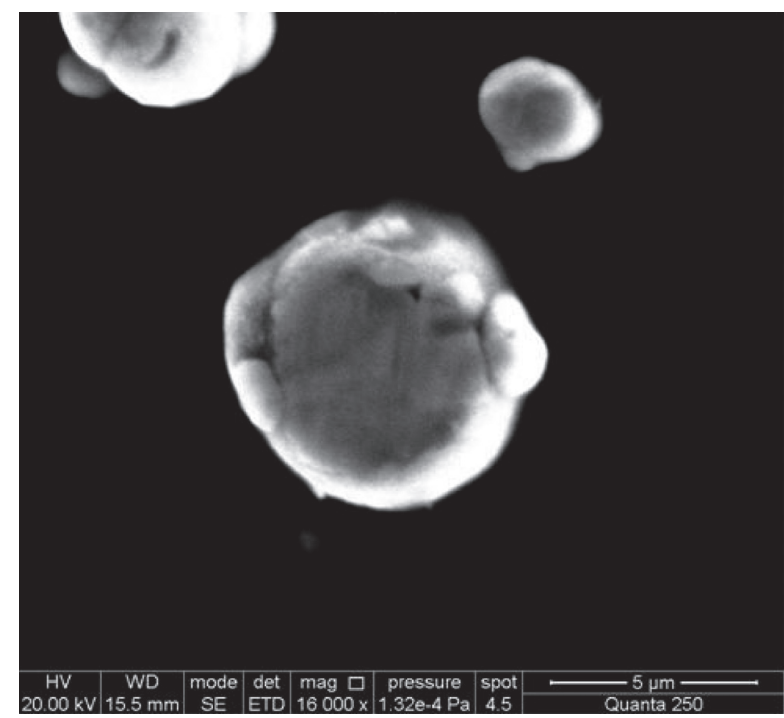

(d)

FIGURe 9: (a) SEM images of magnetic carbonyl iron particles. (b) Modified particles of MRF before experimental test. (c) Multiple particles of MRF after experimental test. (d) Particles of MRF after experimental test. 
In this formula, $h$ is the height of the particle-rich phase relative to $H$, which is the total MR fluid height.

The sedimentation stability curve is shown in Figure 10. It is illustrated that the sedimentation stability of all the samples decreases with time, and at last it remains stable. The sedimentation ratios of samples that experience test are lower than MRF-0, which indicates that the surface of modified particles is damaged by the interactions force between particles during the experiment. In addition, MRF4 has the lowest sedimentation ratio of $73.8 \%$, and it is followed by MRF-2, 74.5\%, MRF-3, 76.1\%, and MRF-1, $78.7 \%$, respectively.

3.3. Zero-Field Viscosity. The experiments of zero-field viscosity are carried out at room temperature, $25^{\circ} \mathrm{C}$, and the rheometer (version: Anton Paar MCR 302) is used in the test of zero-field viscosity. From Figure 11, the zero-field viscosity of all the samples decreases with the increment of shear rate and zero-field viscosity of MRF-0 is lower than that of the others. In addition, MRF-4 has the highest zerofield viscosity. Therefore, MRF subjected to wear experiment has higher zero-field viscosity.

3.4. Shear Yield Stress. The change of maximum transmittable torques of MRF samples with four different drive plates with working time is shown in Figure 12. The maximum transmittable torques of the samples are different. From the maximum to minimum are drive plates with radial groove, pitted surface, smooth surface, and ring groove. The corresponding maximum transmittable torques are $27.7 \mathrm{Nm}, 25 \mathrm{Nm}, 24.4 \mathrm{Nm}$, and $22.3 \mathrm{Nm}$. The rates of decline are $29 \%, 30.6 \%, 30.3 \%$, and $31.2 \%$, respectively. Drive plate with ring groove has the minimum transmit of torque; the reason may be the increase of the working gap of MR fluid as well as the same direction of ring groove and the shear direction of MR fluid, which reduces transmittable torque of MRF.

The relation curves of field current and shear yield stress are obtained in Figure 13. In the working gap between the drive plate and brake plate, the magnetic flux density in $x$ axis direction with different field current is shown in Figure 14. As shown in Figure 13, the shear yield stress of MRF becomes lower after the wear experiment. The curves of MRF-1 and MRF-3 are almost the same, and the shear yield stress reaches $39.7 \mathrm{kPa}$ and $39.8 \mathrm{kPa}$, respectively, when the field current is $4 \mathrm{~A}$. However, MRF-4 has minimum shear yield stress, which is $31.8 \mathrm{kPa}$, when the field current reaches $4 \mathrm{~A}$. Through the above analysis, radial groove and pitted surface drive plates not only improve the transmittable torque of MRF but also increase the wear of MRF.

3.5. Discussion. There are remarkable influences on tribological properties of carbonyl iron-based MRF with different surface topography drive plates. Compared with smooth surface plate, drive plates with radial groove surface and pitted surface can improve transmittable torques of MRF, but drive plate with ring groove surface cannot. However,

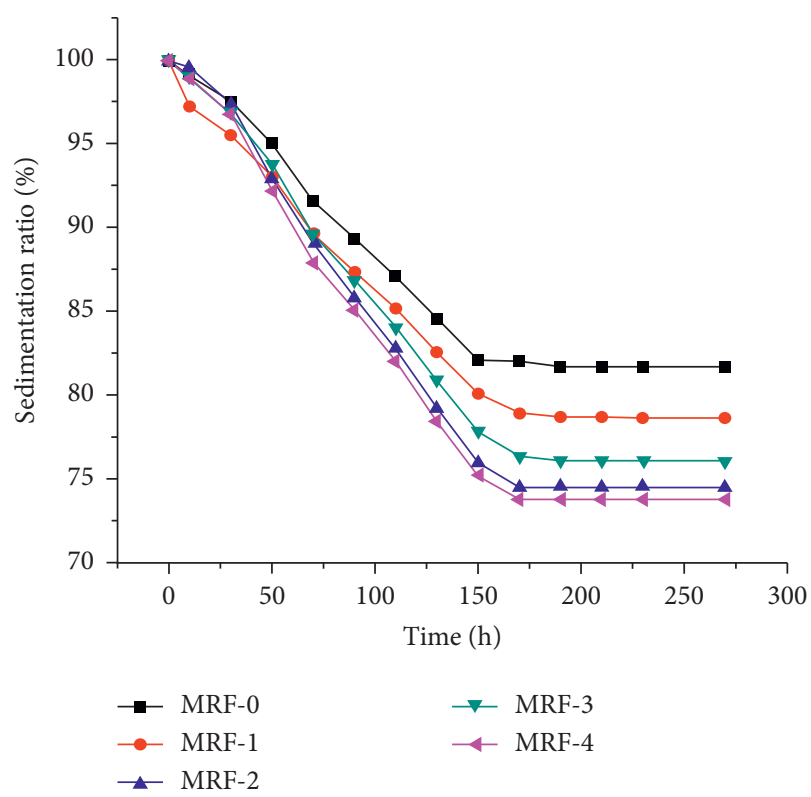

FIGURE 10: Sedimentation ratio of MRF before and after experiments.

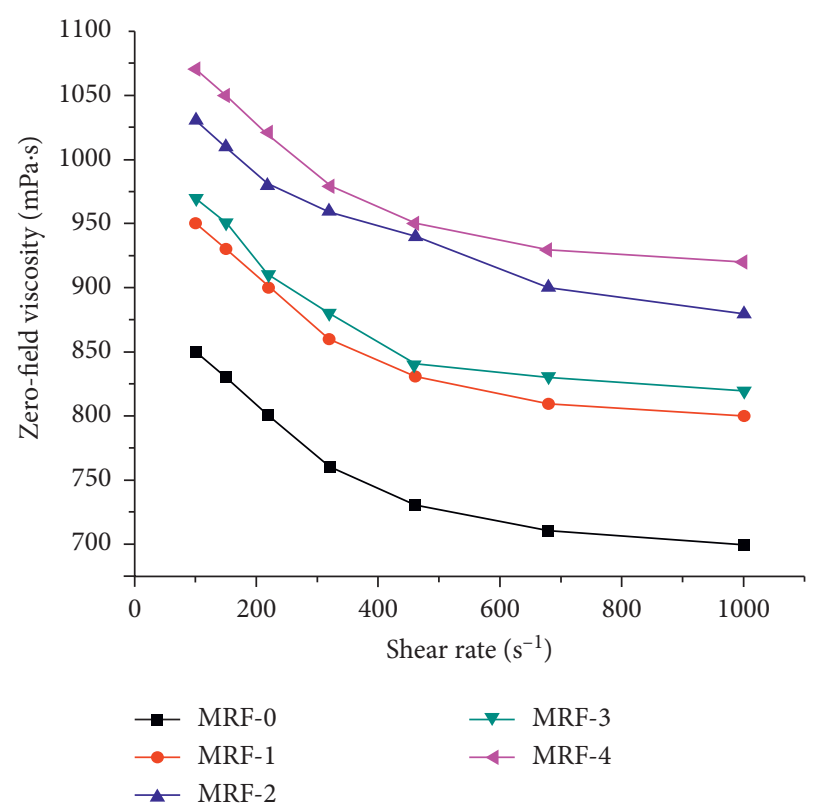

Figure 11: Zero-field viscosity of the samples.

radial groove surface plate and pitted surface plate can also exacerbate the wear of MRF, which will reduce shear yield stress of MRF. In addition, with intensification of the wear of MRF, there results an increase in worn particles, which leads to reduction of zero-field viscosity of MRF. Zero-field viscosity of MRF subjected to wear experiment is as follows in decreasing order: drive plate with radial groove surface, drive plate with pitted surface, drive plate with smooth surface, and drive plate with ring groove surface. Moreover, the sedimentation stability of MRF worsens due to the damage of additives after wear experiments. Therefore, the wear property of MRF needs to be considered in the choice 


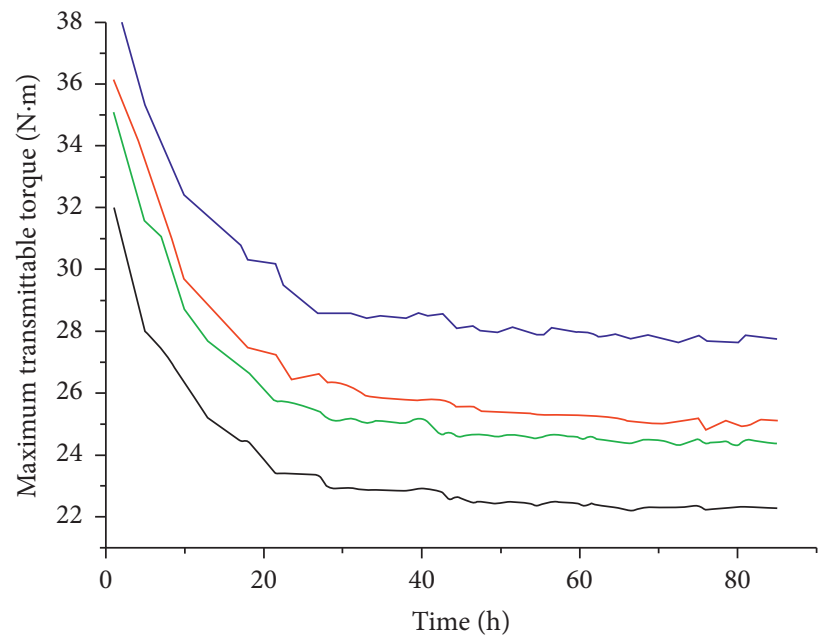

$\begin{array}{ll}\text { MRF-1 } & - \text { MRF-3 } \\ \text { MRF-2 } & \text { MRF-4 }\end{array}$

FIGURE 12: Relationship between maximum transmittable torque of MRF and working time.

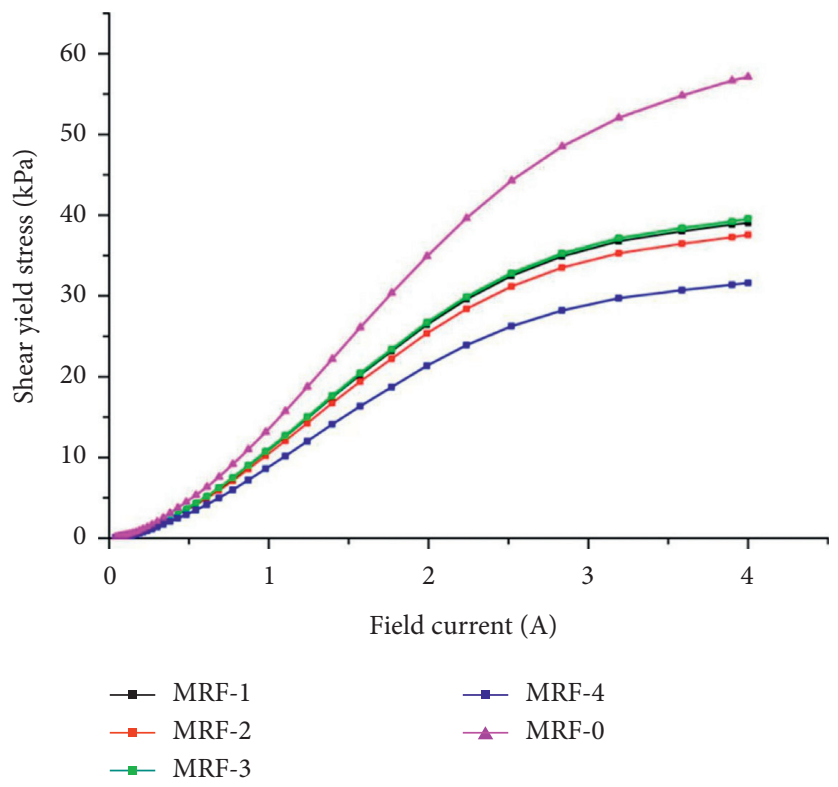

Figure 13: Shear yield stress curves before and after wear experiments.

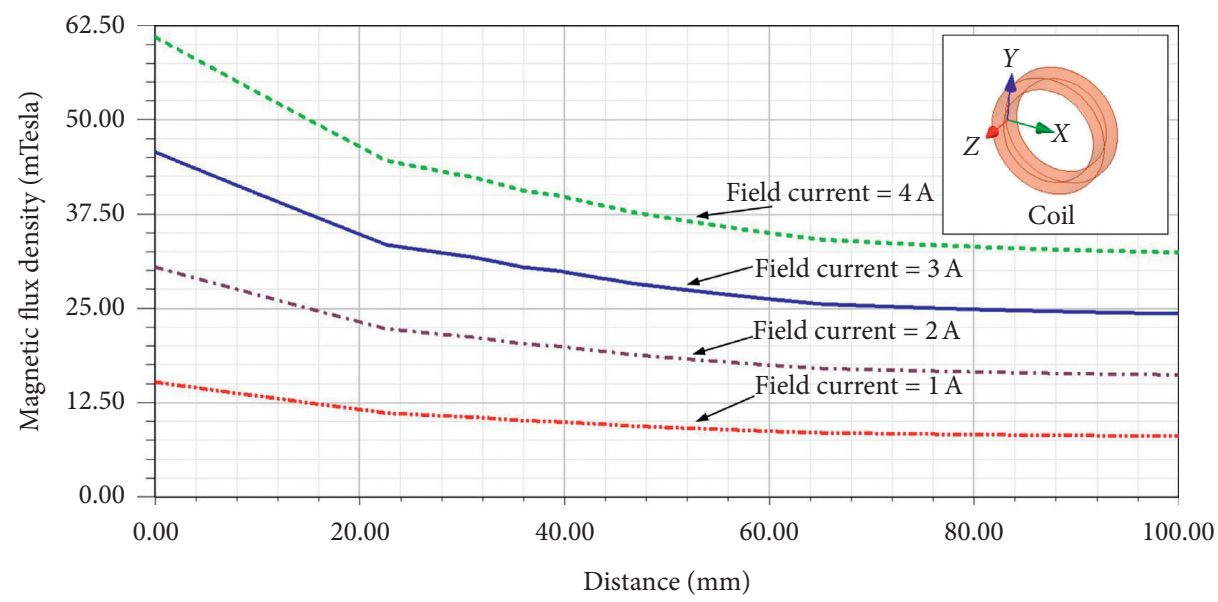

FIgURE 14: Magnetic flux density curves with different field current and position coordinate of coil. 
of surface texture for drive plate applied to MRF transmission system.

\section{Conclusions and Future Work}

A manufacturing process method for MRF was described in detail, drive plates with four different surface topographies were designed, and an experiment table of MRF transmission test-bed was designed and constructed to study the wear property of carbonyl iron-based MRF.

The future work will focus on other factors on the properties of MRF, such as improving control algorithm of MRF transmission system to optimal property of carbonyl iron-based magnetorheological fluid, and the study of the mechanism of friction and wear of MRF is also our future work.

\section{Data Availability}

The data used to support the findings of this study are available from the corresponding author upon request.

\section{Conflicts of Interest}

The authors declare that there are no conflicts of interest regarding the publication of this article.

\section{Acknowledgments}

The support of Qing Lan Project of Colleges and Universities in Jiangsu Province of China, Technology Plan of Lianyungang (no. CG1615), Youth Talents Program (LSZQNXM202001), the National Natural Science Foundation of China (no. 51975568), and the Natural Science Foundation of Jiangsu Province (no. BK20191341) in carrying out this research is gratefully acknowledged.

\section{References}

[1] Y. Gou, C. Sun, Z. Xu, and X. Jing, "Preparation and tests of MR fluids with CI particles coated with MWNTS," Frontiers in Materials, vol. 5, 2018.

[2] F. Chen, H. Li, M. Han, and L. Aimin, "Preparation of magnetorheological fluid with excellent sedimentation stability," Materials and Manufacturing Processes, vol. 35, no. 10, pp. 1077-1083, 2020.

[3] M. Cvek, R. Torres-Mendieta, O. Havelka, M. Urbanek, T. Plachy, and M. Cernik, "Laser-induced fragmentation of carbonyl iron as a clean method to enhance magnetorheological effect," Journal of Cleaner Production, vol. 254, p. 120182, 2020.

[4] W. Zhu, X. Dong, H. Huang, and M. Qi, "Enhanced magnetorheological effect and sedimentation stability of bimodal magnetorheological fluids doped with iron nanoparticles," Journal of Intelligent Material Systems and Structures, 2020.

[5] E. Shokrollahi, A. Goldenberg, J. Eastwood et al., "Application of a nonlinear hammerstein-wiener estimator in the development and control of a magnetorheological fluid haptic device for robotic bone biopsy," Actuators, vol. 7, no. 4, p. 83, 2018 .
[6] A. K. El Wahed, "A novel hydraulic actuation system utilizing magnetorheological fluids for single-port laparoscopic surgery applications," Materials, vol. 13, no. 6, p. 1380, 2020.

[7] R. Desai, S. Acharya, M. Jamadar et al., "Synthesis of magnetorheological fluid and its application in a twin-tube valve mode automotive damper," Proceedings of the Institution of Mechanical Engineers, Part L, vol. 234, no. 7, pp. 1001-1016, 2020.

[8] N. Mutalib, I. Ismail, S. Soffie, and S. N. Aqida, "Magnetorheological finishing on metal surface: a review," IOP Conference Series-Materials Science and Engineering, vol. 469, no. 1, p. 012092, 2019.

[9] B. Kim, J. Chung, M. Cho, and G.-S. Yoon, "Magnetorheological fluid polishing using an electromagnet with straight pole-piece for improving material removal rate," Journal of Mechanical Science and Technology, vol. 32, no. 4, pp. 3345-3350, 2018.

[10] X. Jiang, Y. Zhang, W. Lu, L. Ling, and L. Xiao, "Characteristics of shear stress based on magnetorheological fluid flexible fixture during milling of the thin-walled part," The International Journal of Advanced Manufacturing Technology, vol. 108, no. 7-8, pp. 2607-2619, 2020.

[11] D. Güth and J. Maas, "Long-term stable magnetorheological fluid brake for application in wind turbines," Journal of Intelligent Material Systems and Structures, vol. 27, no. 15, pp. 2125-2142, 2016.

[12] T. Plachy, E. Kutalkova, M. Sedlacik, A. Vesel, M. Masar, and I. Kuritka, "Impact of corrosion process of carbonyl iron particles on magnetorheological behavior of their suspensions," Journal of Industrial and Engineering Chemistry, vol. 66, pp. 362-369, 2018.

[13] M. Sedlacik and V. Pavlinek, "Magnetorheology of dimorphic magnetorheological fluids based on iron nanorods," Journal of Physics: Conference Series, vol. 790, Article ID 012031, 2017.

[14] M. Cvek, M. Mrlik, R. Moucka, and M. Sedlacik, "A systematical study of the overall influence of carbon allotrope additives on performance, stability and redispersibility of magnetorheological fluids," Colloids and Surfaces A: Physicochemical and Engineering Aspects, vol. 543, pp. 83-92, 2018.

[15] M. Ashtiani and S. H. Hashemabadi, "An experimental study on the effect of fatty acid chain length on the magnetorheological fluid stabilization and rheological properties," Colloids and Surfaces A: Physicochemical and Engineering Aspects, vol. 469, pp. 29-35, 2015.

[16] H. Sun, X. Zhu, N. Liu, L. Liang, and L. Shixu, "Effect of different volume fraction magnetorheological fluids on its shear properties," Journal of Physics Conference Series, vol. 1187, no. 3, p. 032078, 2019.

[17] S. T. Shilan, S. A. Mazlan, M. H. A. Khairi, and Ubaidillah, "Properties of plate-like carbonyl iron particle for magnetorheological fluid," Journal of Physics: Conference Series, vol. 776, p. 012033, 2016.

[18] S. Patel, D. Patel, and R. Upadhyay, "Performance enhancement of MR brake using flake-shaped iron-particlebased magnetorheological fluid," Journal of Testing and Evaluation, vol. 48, no. 3, pp. 2393-2411, 2020.

[19] Y. Wang, W. Xie, and D. Wu, "Rheological properties of magnetorheological suspensions stabilized with nanocelluloses," Carbohydrate Polymers, vol. 231, p. 115776, 2020.

[20] M. Zhao, J. Zhang, and J. Yao, "Effects of Nano-Diamond on Magnetorheological Fluid Properties," Nano, vol. 1210 pages, 2017.

[21] W. Zhu, X. Dong, H. Huang, and M. Qi, "Iron nanoparticlesbased magnetorheological fluids: a balance between MR effect 
and sedimentation stability," Journal of Magnetism and Magnetic Materials, vol. 491, p. 165556, 2019.

[22] M. McKee, F. Gordaninejad, and X. Wang, "Effects of temperature on performance of compressible magnetorheological fluid suspension systems," Journal of Intelligent Material Systems and Structures, vol. 29, no. 1, pp. 41-51, 2018.

[23] N. Wang, X. Liu, S. Sun, G. Królczyk, Z. Li, and W. Li, "Microscopic characteristics of magnetorheological fluids subjected to magnetic fields," Journal of Magnetism and Magnetic Materials, vol. 501, p. 166443, 2020.

[24] E. Park, L. da Luz, and A. Suleman, "Multidisciplinary design optimization of an automotive magnetorheological brake design," Computers and Structures, vol. 86, no. 3-5, pp. 207-216, 2008.

[25] N. Wang, D. H. Li, W. L. Song, S. C. Xiu, and X. Z. Meng, "Effect of surface texture and working gap on the braking performance of the magnetorheological fluid brake," Smart Materials and Structures, vol. 25, no. 10, p. 105026, 2016.

[26] Q. Zhang, X. Liu, Y. Ren, L. Wang, and Y. Hu, "Effect of particle size on the wear property of magnetorheological fluid," Advances in Materials Science and Engineering, vol. 2016, Article ID 4740986, 7 pages, 2016.

[27] M. Sedlacik, V. Pavlinek, R. Vyroubal, P. Peer, and P. Filip, “A dimorphic magnetorheological fluid with improved oxidation and chemical stability under oscillatory shear," Smart Materials and Structures, vol. 22, no. 3, Article ID 035011, 2013. 